\title{
Fuzzy Metrics for Solving MODM Problems
}

\author{
Olga Grigorenko \\ Institute of Mathematics and Computer Science, University of Latvia, \\ Raina bulvaris 29, Riga, LV-1459, Latvija, ol .grigorenko@gmail.com
}

\begin{abstract}
This paper presents a solution approach for multi-objective linear programming problem. We consider MOLP problem where optimization of multiple, conflicting objective functions subject to constraints is concerned. We give also comments how to deal with FMOLP problem, where the parameters are imprecisely or ambiguously known to the experts. Fuzzy approach for solving MOLP problem is one of the techniques most frequently used in the literature. We propose to involve fuzzy metrics to describe the objective functions where in "classical" fuzzy approach the membership functions which illustrate how far the concrete point is from the solution of individual problem are studied.
\end{abstract}

Keywords: Multi-objective linear programming, Fuzzy metrics, Fuzzy relations, Multiobjective decision making, Aggregation of fuzzy metrics.

\section{Introduction}

Multi-objective decision making (MODM) is a modelling and methodological tool for dealing with complex engineering, production planning, logistics, environment management, banking/finance planning and many others problems. Decision makers face a problem that different objectives conflict one with another, also other information could be incomplete and/or vague. Fuzzy set approaches are suitable and helpful when dealing with uncertainty and conflict in processes. In multiple objective decision making, application functions (membership functions or fuzzy sets) are established to measure the degree of fulfillment of the decision maker's requirements (achievement of goals, nearness to an ideal point, satisfaction, etc.) for the objective functions and are used in the process of finding "good compromise" solution. MODM problems can be categorized in different ways, such as by the class of the model (e.g., linear, nonlinear, or stochastic), by the characteristic of the decision space (e.g., finite or infinite), or by the nature of solution process (e.g., prior specification of preferences or interactive). Here we review and analyse classical multiple-objective decision making methods and present our approach where we involve fuzzy metrics to describe the distance to the solutions of individual problems where in classical fuzzy approach the membership functions (fuzzy sets) which illustrate how far the concrete point is from the solution of individual problem are studied. The important difference between two approaches is that if we measure distance only to the solution of individual problem (using fuzzy set) we forget about the triangular inequality of distance function and after an aggregation process it could lead to the loss of Pareto optimal solution. By using fuzzy metrics we solve this problem.

We consider the following multiple objective linear programming problem:

$M A X Z$, where $Z=\left(z_{1}, \ldots, z_{k}\right)$ is a vector of objectives, $z_{i}=\sum_{j=1}^{n} c_{i j} x_{j}$, where $i=1, . ., k$, subject to $\sum_{j=1}^{n} a_{i j} x_{j} \leq b_{i}$, $i=1, \ldots, m$;

That is we must find a vector $\mathbf{x}^{\mathbf{0}}=\left(x_{1}^{o}, \ldots, x_{n}^{o}\right)$, which maximizes $k$ objective functions of $n$ variables, and with $m$ constraints. Let $D$ denote a feasible region of the problem (1).

For the sake of brevity further we denote vectors in bold e.g. $\mathbf{x}=\left(x_{1}, \ldots, x_{n}\right), \mathbf{y}^{*}=\left(y_{1}^{*}, \ldots, y_{n}^{*}\right)$.

In problem (1), all objective functions can hardly reach their optima at the same time subject to the given constraints since usually objective functions conflict with one another. Thus Pareto optimal solution (efficient solution) and optimal compromise solution are intro- 
duced:

Definition 1.1 [16] $\mathbf{x}^{*}$ is called Pareto optimal solution if and only if there does not exist another $\mathbf{x} \in D$ such that $z_{i}\left(\mathbf{x}^{*}\right) \leq z_{i}(\mathbf{x})$ for all $i$ and $z_{j}\left(\mathbf{x}^{*}\right) \neq z_{j}(\mathbf{x})$ for at least one $j$.

Definition 1.2 [16] An optimal compromise solution of a vector-maximum problem is a solution $\mathbf{x} \in D$ which is preferred by the decision maker to all other solutions, taking into consideration all criteria contained in the vector-valued objective function. It is generally accepted, that an optimal compromise solution has to be a Pareto optimal solution. Further we will call optimal compromise solution simply optimal solution.

Thus our main aim is to determine the optimal compromise solution. The fuzzy approach for solving MOLP proposed by Zimmermann [15] has given an effective way of measuring the satisfaction degree for MOLP. The idea is to identify the membership functions prescribing the fuzzy goals (solutions of individual problem) for the objective functions $z_{i}, i=1, \ldots, k$. The following linear function is an example of a membership function:

$$
\mu_{i}(\mathbf{x})= \begin{cases}0, & \text { if } z_{i}(\mathbf{x})<z_{i}^{\min } \\ \frac{z_{i}(\mathbf{x})-z_{i}^{\min }}{z_{i}^{\max }-z_{i}^{\min }}, & z_{i}^{\min } \leq z_{i}(\mathbf{x}) \leq z_{i}^{\max }, \\ 1, & z_{i}(\mathbf{x})>z_{i}^{\max }\end{cases}
$$

where $\mathbf{x}_{\mathbf{i}}{ }^{\max }$ is the solution of individual problem

$\operatorname{MAX} z_{i}$, s.t. $\sum_{j=1}^{n} a_{i j} x_{j} \leq b_{i}, i=1, \ldots, m$ and

$z_{i}^{\max }=z_{i}\left(\mathbf{x}_{\mathbf{i}}^{\max }\right)$;

$\mathbf{x}_{\mathbf{i}}^{\text {min }}$ is the solution of individual problem

$M I N z_{i}$, s.t. $\sum_{j=1}^{n} a_{i j} x_{j} \leq b_{i}, i=1, \ldots, m$

and $z_{i}^{\min }=z_{i}\left(\mathbf{x}_{\mathbf{i}}^{\text {min }}\right)$.

Further in the "classical" fuzzy approach membership functions $\mu_{i}$ are aggregated. The main subject which is discussed is the choice of an aggregation function and if the Pareto optimal solution is obtained after the aggregation process.

In our paper we propose a completely different approach although we still work the fuzzy environment.

We initiate involving of fuzzy metrics to solve the problem. To justify the choice of fuzzy metrics let us first observe the classical linear programming problem when we should maximize the unique function $z=\sum_{j=1}^{n} c_{j} x_{j}$ where the vectors $\left(x_{1}, \ldots, x_{n}\right)$ belong to the set $D: \sum_{j=1}^{n} a_{i j} x_{j} \leq b_{i}, i=1, \ldots, m$. And let $\mathbf{x}^{\max }$ be a so- lution of the problem. In this case we can involve the metric $d$ :

$$
d(\mathbf{x}, \mathbf{y})=|z(\mathbf{x})-z(\mathbf{y})|
$$

which is obviously a crisp metric.

Thus we can reformulate the problem in the following way:

$\operatorname{MIN} m(\mathbf{x})=d\left(\mathbf{x}, \mathbf{x}^{\max }\right)$, where the vectors $\left(x_{1}, \ldots, x_{n}\right)$ belong to the set $D$. That is we should find a vector $\mathbf{x} \in$ $D$ for which the function $m$ takes the minimum value.

We use this idea to solve the multi-objective linear programming problem. Since we have more than one objective function we should involve metrics for each objective function and they should be obviously fuzzy metrics to overcome the conflict of all objective functions. Further we aggregate fuzzy metrics to get one fuzzy metric which include the information about all objective functions and in the last step we should find a vector from the set $D$ which has minimal distance to all individual solutions (with respect to the aggregated fuzzy metric). Thus the scheme of finding solution is as follows:

1. We define fuzzy metrics $M_{i}$ which generalize the following crisp metrics:

$$
d_{i}(\mathbf{x}, \mathbf{y})=\left|z_{i}(\mathbf{x})-z_{i}(\mathbf{y})\right|, i=1, . ., k .
$$

Thus each fuzzy metric describes corresponding objective function $z_{i}$.

2. We aggregate fuzzy metrics using an aggregation function $A$ which preserves the properties of initial fuzzy metrics.

Thus the aggregated fuzzy metric provides the information about all objective functions.

3. We find a minimum in the set $D$ with respect to the aggregated fuzzy metric.

In our work we exactly realize the above described scheme. As we have seen above, solving the classical linear programming problem with one objective function there is naturally arisen metric, which could be naturally generalized to fuzzy metric. Thus if we use fuzzy approach proposed by Zimmermann [15] and generalized by many others authors (see e.g. [12], [13], [6]) we do not take into account the information about these metrics, so this information is lost. The most important is the triangular inequality. Thus one of the advantages of our approach is that we take into account this information, and even more aggregating these fuzzy metrics we use the aggregation function which preserve the properties of initial fuzzy metrics. The other advantage is that in our approach we explain 
the choice of aggregation function (this is caused by the fuzzy environment (or t-norm) in which we are working). Moreover in our approach we can naturally use compensatory aggregation functions and even more we can use weights to show the preference of objective functions.

As we wrote in Definition 2, an optimal compromise solution has to be a Pareto optimal solution.As we will see later, in our approach we have found the properties which guarantee Pareto-optimality even regardless of the uniqueness of the optimal solution.

This approach is an extension of the approach presented in [5].

\section{Preliminaries}

Definition 2.1 (see e.g. [2]) A fuzzy binary relation $E$ on a set $X$ is called a fuzzy equivalence relation with respect to a t-norm $T$ (or $T$-equivalence, or $T$ similarity relation), if and only if the following three axioms are fulfilled for all $x, y, z \in X$ :

1. $E(x, x)=1$ reflexivity;

2. $E(x, y)=E(y, x)$ symmetry;

3. $T(E(x, y), E(y, z)) \leq E(x, z) T$-transitivity.

The following result establishes principles of construction of fuzzy equivalence relations using pseudometrics.

Theorem 2.1 ([1]) Let $T$ be a continuous Archimedean t-norm with an additive generator t. For any pseudo-metric $d$, the mapping

$$
E_{d}(x, y)=t^{(-1)}(\min (d(x, y), t(0)))
$$

is a $T$-equivalence.

Example 2.1 Let us consider the set of real numbers $X=\mathbb{R}$ and metric $d(x, y)=|x-y|$ on it. Taking into account that $t_{L}(x)=1-x$ is an additive generator of $T_{L}$ (Eukasiewicz $t$-norm) and that $t_{P}(x)=-\ln (x)$ is an additive generator of $T_{P}$ (product t-norm), we obtain two fuzzy equivalence relations:

$$
\begin{gathered}
E_{L}(x, y)=\max (1-|x-y|, 0) ; \\
E_{P}(x, y)=e^{-|x-y|} .
\end{gathered}
$$

\section{Fuzzy metric}

Nowadays in literature the notion of fuzzy metric is basically used under the axioms introduced in [3] and [?]. These axioms are actually a reformulation of the axioms originally defined in [9]. In [9] the idea for definition of fuzzy metric comes from the assertion that the considered value of a crisp metric $d(x, y)$ (which should by fuzzified or approximated) is smaller than an priori given real number $\lambda$. In other words the statement $d(x, y)<\lambda$ is fuzzified. We propose to define fuzzy metric as the degree to which the observed crisp metric of $x$ and $y(d(x, y))$ is equal to the real number $\lambda$, equal in fuzzy sense. That is we define fuzzy metric (or $E$ - $d$-metric ) as a mapping

$$
M_{E}: X^{2} \times[0, \infty) \rightarrow[0,1]:
$$

Definition 3.1 Let $d$ be a crisp metric, $t \in[0, \infty)$ and $E$ is a fuzzy equivalence. Then a mapping $M_{E}: X^{2} \times$ $[0, \infty) \rightarrow[0,1]$

$$
M_{E}(x, y, t)=E(d(x, y), t),
$$

is called a fuzzy metric or E-d-metric.

Example 3.1 Let $d$ be a crisp metric, $t \in[0, \infty)$. Then we have the following two examples of fuzzy metrics:

$$
\begin{aligned}
& \text { - } M_{E_{L}}(x, y, t)=E_{L}(d(x, y), t)= \\
& =\max (1-|d(x, y)-t|, 0) \\
& \text { - } M_{E_{P}}(x, y, t)=E_{P}(d(x, y), t)=e^{-|d(x, y)-t|} .
\end{aligned}
$$

Thus for the upper defined metrics for our solution approach we have the following fuzzy metrics:

Example 3.2 Let $t \in[0, \infty)$ and $\mathbf{x}, \mathbf{y} \in D$. Then we have the following two examples of fuzzy metrics, which we will use in our solution approach:

$$
\begin{aligned}
& \cdot M_{E_{L}}(\mathbf{x}, \mathbf{y}, t)=E_{L}\left(\left|z_{i}(\mathbf{x})-z_{i}(\mathbf{y})\right|, t\right)= \\
& =\max \left(1-|| z_{i}(\mathbf{x})-z_{i}(\mathbf{y})|-t|, 0\right) \\
& \cdot M_{E_{P}}(\mathbf{x}, \mathbf{y}, t)=E_{P}\left(\left|z_{i}(\mathbf{x})-z_{i}(\mathbf{y})\right|, t\right)= \\
& e^{-|| z_{i}(\mathbf{x})-z_{i}(\mathbf{y})|-t|} .
\end{aligned}
$$

Now let us formulate axioms for fuzzy metric $M$, not using crisp metric $d$. But we still want to fuzzify the statement $d(x, y)=\lambda$, where $\lambda$ is an priory given real number. Thus:

Definition 3.2 Let $T$ be a $t$-norm and $t \in[0, \infty)$. Then a mapping $M: X^{2} \times[0, \infty) \rightarrow[0,1]$ is called fuzzy metric if it satisfies the following properties:
1. $M(x, y, 0)=1$ if and only if $x=y$;
2. $M(x, y, t)=M(y, x, t)$;
3. $T(M(x, y, t), M(y, z, s)) \leq M(x, z, t+s)$ 
If the first two axioms are clear then the third one arises from the statement:

$d(x, y)=t$ and $d(y, z)=s$ then $d(x, z) \leq t+s$. Which is in fuzzy language $T(E(d(x, y), t), E((y, z), s)) \leq$ $P(d(x, z), t+s)$; which in turn follows: $T(E(d(x, y), t), E((y, z), s)) \leq E(d(x, z), t+s)$.

Theorem 3.1 Let $\left(\mathbb{R}, d^{\prime}\right)$ be a metric space and let $T$ be a continuous Archimedean t-norm with an additive generator $t$. And let

$$
E(x, y)=g^{(-1)}\left(\min \left(d^{\prime}(x, y), g(0)\right)\right)
$$

be a T-equivalence which separates points, then

$$
M(x, y, t)=E(d(x, y), t)
$$

satisfies axioms from the Definition 3.2. for any crisp metric $d$ on $X$.

The fuzzy metrics are constructed (for example Example 3.2) and we come to the next step where we aggregate corresponding metrics.

\section{Aggregation of fuzzy metrics}

The idea of the following section is that we have to fuse the information about all fuzzy metrics $M_{i}=$ $E\left(d_{i}(x, y), t\right)$ and get a global fuzzy metric $M$ which includes the information about all fuzzy metrics $M_{i}$ and thereby also the information about all objective functions $z_{i}$. Actually different $T$-equivalences $E_{i}$ could be used for different functions $z_{i}$, the $T$-norm should be the same. Let us introduce the following mapping $A:[0,1]^{k} \rightarrow[0,1]$ which aggregates fuzzy metrics:

$$
M(\mathbf{x}, \mathbf{y}, t)=A\left(M_{1}(\mathbf{x}, \mathbf{y}, t), \ldots, M_{k}(\mathbf{x}, \mathbf{y}, t)\right) .
$$

It is natural to require from $A$ at least the following properties:

1. If $M_{i}(\mathbf{x}, \mathbf{y}, t)=1$ for all $i$ the global degree should be also 1 . In other words: $A(1, \ldots, 1)=1$.

2. If $M_{i}(\mathbf{x}, \mathbf{y}, t)=0$ for all $i$ the global degree should be also 0 . In other words: $A(0, \ldots, 0)=0$.

3. If one degree $M_{i}(\mathbf{x}, \mathbf{y}, t)$ increases while the others are kept constant, the overall degree must not decrease, i.e. $A$ should be non-decreasing in each component.

That is exactly the definition of aggregation function:

Definition 4.1 [4] An aggregation function is a mapping $A:[0,1]^{k} \rightarrow[0,1]$ which fulfills the following properties:
- $A\left(x_{1}, \ldots, x_{k}\right) \leq A\left(y_{1}, \ldots, y_{k}\right)$ whenever $x_{i} \leq y_{i}$ for all $i \in\{1, \ldots, k\}$ (monotonicity);

- $A(0, \ldots, 0)=0$ and $A(1, \ldots, 1)=1$ (boundary conditions).

For more information about aggregation functions or aggregation operators see [4] and [10].

It is also natural to require that the global fuzzy metric should fulfill the same properties as the individual fuzzy metrics.

Definition 4.2 [14] Consider an n-argument aggregation function $A:[0,1]^{n} \rightarrow[0,1]$ and a $t$-norm $T$. We say that $A$ dominates $T$ iffor all $x_{i} \in[0,1]$ with $i \in\{1, \ldots, n\}$ and $y_{i} \in[0,1]$ with $i \in\{1, \ldots, n\}$ the following property holds:

$$
\begin{gathered}
T\left(A\left(x_{1}, \ldots, x_{n}\right), A\left(y_{1}, \ldots, y_{n}\right)\right) \leq \\
\leq A\left(T\left(x_{1}, y_{1}\right), \ldots, T\left(x_{n}, y_{n}\right)\right) .
\end{gathered}
$$

Theorem 4.1 Let $|X|>3$ and let $T$ be $a t$ norm. If $M_{i}$ for all $i \in\{1, \ldots, n\}$ are fuzzy metrics (with respect to the $t$-norm $T)$; then $M(x, y, t)=$ $A\left(M_{1}(x, y, t), \ldots, M_{n}(x, y, t)\right)$ is fuzzy metrics (with respect to the t-norm $T$ ) if A belongs to the class of aggregation functions which dominate $T$ and $A$ is strictly monotone function.

Proof:

1. Since for all $i M_{i}(x, y, 0)=1$ if and only if $x=y$

$$
A\left(M_{1}(x, y, 0), \ldots, M_{n}(x, y, 0)\right)=1
$$

if and only if $x=y$.

2. $T(M(x, y, t), M(y, z, s)) \leq M(x, z, t+s)$ :

$$
\begin{gathered}
T(M(x, y, t), M(y, z, s))= \\
=T\left(A\left(M_{1}(x, y, t), \ldots, M_{n}(x, y, t)\right),\right. \\
\left.A\left(M_{1}(y, z, s), \ldots, M_{n}(y, z, s)\right)\right) \leq \\
\leq A\left(T\left(M_{1}(x, y, t), M_{1}(y, z, s)\right), \ldots,\right. \\
\left.T\left(M_{n}(x, y, t), M_{n}(y, z, t)\right)\right)
\end{gathered}
$$

because of the dominance of $T$ by A. Further

$$
\begin{gathered}
A\left(T\left(M_{1}(x, y, t), M_{1}(y, z, s)\right), \ldots,\right. \\
\left.T\left(M_{n}(x, y, t), M_{n}(y, z, t)\right)\right) \leq \\
\leq A\left(M_{1}(x, z, t+s), \ldots, M_{n}(x, z, t+s)\right)
\end{gathered}
$$

since $A$ is a monotone function and $T\left(M_{i}(x, y, t), M_{i}(y, z, s)\right) \leq M_{i}(x, z, t+s)$ for all $i$. Thus we have proven the required inequality. 
It was important to find conditions for an aggregation function which guarantee the preservation of the properties of fuzzy metric in the aggregation process.

In the next two examples we observe the aggregation function which dominates Łukasiewicz and product tnorms:

Example 4.1 [14] For any $k>2$ and any $p=$ $\left(p_{1}, \ldots, p_{k}\right)$ with $\sum_{i=1}^{k} p_{i} \geq 1$ and $p_{i} \in[0, \infty] k$-ary aggregation function

$$
A_{p}\left(x_{1}, \ldots, x_{k}\right)=\max \left(\sum_{i=1}^{k} x_{i} \cdot p_{i}+1-\sum_{i=1}^{k} p_{i}, 0\right)
$$

dominates Łukasiewicz t-norm $T_{L}$.

Example 4.2 [14] For any $k>2$ and any $p=$ $\left(p_{1}, \ldots, p_{k}\right)$ with $\sum_{i=1}^{k} p_{i} \geq 1$ and $p_{i} \in[0, \infty] k$-ary aggregation function

$$
A_{p}\left(x_{1}, \ldots, x_{k}\right)=\prod_{i=1}^{k} x_{i}^{p_{i}}
$$

dominates product t-norm $T_{P}$.

\section{Solution approach}

Let us denote the function $m: X \times[0, \infty) \rightarrow[0,1]$ by:

$$
m(\mathbf{x}, t)=M\left(\mathbf{x}, \mathbf{x}_{\mathbf{1}}, t\right) \wedge M\left(\mathbf{x}, \mathbf{x}_{\mathbf{2}}, t\right) \wedge \ldots \wedge M\left(\mathbf{x}, \mathbf{x}_{\mathbf{k}}, t\right) .
$$

This means that we find for each $\mathbf{x} \in D$ the value $M\left(\mathbf{x}, \mathbf{x}_{1}, t\right) \wedge M\left(\mathbf{x}, \mathbf{x}_{\mathbf{2}}, t\right) \wedge \ldots \wedge M\left(\mathbf{x}, \mathbf{x}_{\mathbf{k}}, t\right)$, that is we find the degree to which distance between $\mathbf{x}$ and any of $\mathbf{x}_{\mathbf{k}}$ is $t$, where $\mathbf{x}_{\mathbf{k}}$ are solutions of individual problems. That is analogue of the function $m$ described in Section 1. This function shows the distance from point $\mathbf{x}$ to the optimal solution, but in fuzzy sense. Since the distance here is $t$ and $m(\mathbf{x}, t)$ shows the degree to which distance from point $\mathbf{x}$ to the optimal solution is $t$ we should find $\mathbf{x}$ for which $t$ is minimal and $m(\mathbf{x}, t)$ is maximal. That is, for each $\mathbf{x}$ we find $t$ for which value $m(\mathbf{x}, t)$ is maximal. Thus we solve the following problem: $\operatorname{MAXm}_{x}(t)$, s.t.t $\in[0, \infty)$, and so for every $\mathbf{x}$ we have $t$ for which $m(\mathbf{x}, t)$ is maximal. Then we choose $\mathbf{x}$ for which $t$ is minimal.

Thus the multi-objective linear programming problem comes to the following problem:

$$
\min _{\mathbf{x}} \max _{\mathbf{t}} M\left(\mathbf{x}, \mathbf{x}_{\mathbf{1}}, t\right) \wedge M\left(\mathbf{x}, \mathbf{x}_{\mathbf{2}}, t\right) \wedge \ldots \wedge M\left(\mathbf{x}, \mathbf{x}_{\mathbf{k}}, t\right)
$$

Theorem 5.1 An optimal solution $\mathbf{x}$ to the problem $(P)$ is a Pareto optimal solution if it is the unique optimal solution.

Proof:

If $\mathbf{x}$ is not a Pareto optimal solution then there exists another $\tilde{\mathbf{x}} \in D$ such that $z_{i}(\mathbf{x}) \leq z_{i}(\tilde{\mathbf{x}})$ for all $i$ and $z_{j}(\mathbf{x}) \neq z_{j}(\tilde{\mathbf{x}})$ for at least one j. That means that $d_{j}\left(\tilde{\mathbf{x}}, \mathbf{x}_{\mathbf{j}}\right)<d_{j}\left(\mathbf{x}, \mathbf{x}_{\mathbf{j}}\right)$ and $\left.d_{i}(\tilde{\mathbf{x}}, \mathbf{x}) \leq \mathbf{d}_{(\mathbf{x}}, \mathbf{x}_{\mathbf{i}}\right)$ for any other $i$. Let us now compare $M\left(\mathbf{x}, \mathbf{x}_{\mathbf{1}}, t\right) \wedge M\left(\mathbf{x}, \mathbf{x}_{\mathbf{2}}, t\right) \wedge \ldots \wedge M\left(\mathbf{x}, \mathbf{x}_{\mathbf{k}}, t\right)$ and $M\left(\tilde{\mathbf{x}}, \mathbf{x}_{1}, t\right) \wedge M\left(\tilde{\mathbf{x}}, \mathbf{x}_{\mathbf{2}}, t\right) \wedge \ldots \wedge M\left(\tilde{\mathbf{x}}, \mathbf{x}_{\mathbf{k}}, t\right)$ : $\max _{\mathbf{t}} M\left(\tilde{\mathbf{x}}, \mathbf{x}_{\mathbf{1}}, t\right) \wedge M\left(\tilde{\mathbf{x}}, \mathbf{x}_{\mathbf{2}}, t\right) \wedge \ldots \wedge M\left(\tilde{\mathbf{x}}, \mathbf{x}_{\mathbf{k}}, t\right) \leq$ $\leq \max _{\mathbf{t}} M\left(\mathbf{x}, \mathbf{x}_{\mathbf{1}}, t\right) \wedge M\left(\mathbf{x}, \mathbf{x}_{\mathbf{2}}, t\right) \wedge \ldots \wedge M\left(\mathbf{x}, \mathbf{x}_{\mathbf{k}}, t\right)$. This contradicts the fact that $\mathbf{x}$ is the unique optimal solution to the problem.

We can also prove the above theorem without demanding the "uniqueness of the optimal solution" but in this case we should require some specific properties:

Theorem 5.2 An optimal solution $\mathbf{x}$ to the problem $(P)$ is a Pareto optimal solution if fuzzy metrics $M_{i}$ are built with respect to the fuzzy equivalences which separate points and $A$ is a strictly monotone function.

These properties are quite natural ones since by this we simply require that the metric $M$ should react to any change of any of the functions $z_{i}$. Thus for practical applications we suggest to use fuzzy metrics and aggregation functions respecting these properties.

\section{Solution approach for FMOLP problem}

Let us observe MOLP problem (1). It is natural to recognize that the possible values of the parameters $c_{i j}$ are often imprecisely or ambiguously known to the experts. In this case, it may be more appropriate to interpret the experts' understanding of the parameters as fuzzy numerical data that can be represented by fuzzy numbers. We propose to use extensional fuzzy numbers.

Definition 6.1 ([8], [7]) Let $E$ be a T-equivalence relation on $\mathbb{R}$ and $x \in \mathbb{R}$ (a real number which should be fuzzyfied). Then we define the extensional fuzzy num$\operatorname{ber} x_{E}\left(x_{E}: \mathbb{R} \rightarrow[0,1]\right)$ by:

$$
x_{E}(y)=E(x, y)
$$

The following operations with extensional fuzzy numbers are defined in [7]:

- $x_{E}+y_{E}=(x+y)_{E}$ 


$$
\begin{aligned}
& -x_{E} \cdot y_{E}=(x \cdot y)_{E} \\
& \text { - } x_{E}+y_{D}=(x+y)_{\max (E, D)} \\
& \text { - } x_{E} \cdot y_{D}=(x \cdot y)_{\max (E, D)}
\end{aligned}
$$

Thus for function (linear function) with coefficients as fuzzy extensional numbers the value for the any argument $\mathbf{x}$ is a fuzzy extensional number. For example for function $z_{i}(\mathbf{x})=\sum_{j=1}^{n} c_{i j_{E}} x_{j}$ (where $c_{i j_{E}}$ are fuzzy extensional number, $E$ is an equivalence relation) the value $z_{i}(\mathbf{x})$ is calculated as: $\left(\sum_{j=1}^{n} c_{i j} x_{j}\right)_{E}$. It is also possible to use different $T$-equivalences.

The following metric is involved in [7]:

$\left|x_{E}-y_{D}\right|=|x-y|_{\max (E, D)}$

or another crisp metric could be used:

$d\left(x_{E}, y_{D}\right)=d(x, y)_{\max (E, D)}$. The above metric is a fuzzy metric if we define fuzzy metric as $M(x, y, t)=$ $E(d(x, y), t)$, where $d(x, y)$ is a crisp metric and $E$ an equivalence relation. And

$$
M(x, y, t)=\left\{\begin{array}{l}
1, \text { if } t>d(x, y) \\
d\left(x_{E}-y_{D}\right), \text { otherwise }
\end{array}\right.
$$

is a fuzzy metric according to the "classical" fuzzy metric definition if $E$ and $D$ are $T$-equivalence relations, where $T$ is a continuous t-norm with additive generator. It follows from the $d\left(x_{E}, y_{E}\right)$ definition and theorem 3.1. [11]

Let us consider the fuzzy metric $d_{S}$, where $S$ is an equivalence relation and let $T$ be a continuous Archimedean $t$-norm with an additive generator $t$. We build a new fuzzy equivalence relation $E_{d_{S}}: X \times X \rightarrow F$ where for each $x$ and $y$ we have a fuzzy set using construction of Theorem 3.1. The fuzzy equivalence relations $E_{d_{S}}$ is a fuzzy metric. Thus for each objective function $z_{i}$ (with parameters as fuzzy extensional numbers $c_{i j E_{i j}}$ ) we have:

$$
\begin{aligned}
& \text { 1. } d_{i}(\mathbf{x}, \mathbf{y})=\left|z_{i}(\mathbf{x})-z_{i}(\mathbf{y})\right| \\
& \text { 2. } d_{i}(\mathbf{x}, \mathbf{y})_{S_{i}}=\left|\sum_{j=1}^{n} c_{i j} x_{j}-\sum_{j=1}^{n} c_{i j} x_{j}\right|_{\max _{j} E_{i j}} \\
& \text { 3. } M_{i}(\mathbf{x}, \mathbf{y}, t)=E_{d_{S_{i}}}(\mathbf{x}, \mathbf{y})(t)
\end{aligned}
$$

Further we realize the upper described scheme for MOLP problem.

\section{Conclusions}

In our paper we proposed a solution approach for multi-objective linear programming problem where we have used fuzzy metrics instead of the membership functions prescribing the satisfaction degree of reaching the solution of individual problems. Further, to get an optimal compromise solution the fuzzy metrics were aggregated and the "minimum" with respect to the aggregated fuzzy metric has been found. Although the approach described in our paper is more complicated in computations it has the following advantages:

1. This approach generalizes the classical linear programming approach.

2. There is a reasonable explanation of the choice of aggregation operator.

3. The compensatory aggregation operator can be easily applied.

4. The Pareto optimal solution is received.

We gave also some comments how to solve FMOLP problem.

Some important themes are discussed in the paper besides the solution approach, but which were significant for it: different definitions of fuzzy metrics and connection between them were observed, aggregation of fuzzy metrics were studied.

\section{Acknowledgement}

The work has been supported by European Regional Development Fund within the project Nr.1.1.1.2/16/I/001, application Nr.1.1.1.2/VIAA/4/20/707 "Fuzzy relations and fuzzy metrics for customer behavior modeling and analyis".

\section{References}

[1] B. D. Baets, R. Mesiar, Pseudo-metrics and $t$ equivalences, J. Fuzzy Math 5 (1997) 471-481.

[2] U. Bodenhofer, A similarity-based generalization of fuzzy orderings preserving the classical axioms, Internat. J. Uncertain. Fuzziness Knowledge-Based Systems 8 (5) (2000) 593610.

[3] A. George, P. Veeramani, On some results in fuzzy metric spaces, Fuzzy Sets and Systems 64 (1994) 395-399. 
[4] M. Grabisch, J.-L. Marichal, R. Mesiar, E. Pap, Aggregation Functions (Encyclopedia of Mathematics and its Applications), Cambridge University Press, UK, 2009.

[5] O. Grigorenko, Involving fuzzy orders for multi-objective linear programming, Mathematical Modelling and Analysis 17(3) (2012) 366382.

[6] S.-M. Guu, Y.-K. Wu, Fuzzy sets and systems, Weighted coefficients in two-phase approach for solving the multiple objective programmin gproblems 85 (1997) 45-48.

[7] M. Holčapek, M. Štěpnička, Mi-algebras: A new framework for arithmetics of (extensional) fuzzy numbers, Fuzzy Sets and Systems 257 (2014) 102-131.

[8] F. Klawonn, Fuzzy points, fuzzy relations and fuzzy functions, in: V. Novák, I. Perfilieva (Eds.), Discovering the World with Fuzzy Logic, Springer, Berlin, 2000, pp. 431-453.

[9] I. Kramosil, J. Michalek, Fuzzy metrics and statistical metric spaces, Kybernetika 11 (1975) 336-344.

[10] R. Mesiar, T. Calvo, G. Mayor, Aggregation Operators: New Trends and Applications (Studies in Fuzziness and Soft Computing), Physica-Verlag, Heidelberg, New York, 2002.

[11] J. Miñana, O. Valero, A duality relationship between fuzzy metrics and metrics, International Journal of General Systems 47(6) (2018) 593612.

[12] M. Sakawa, H. Yano, Interactive decision making for multiple non linear programming using augmented minimax problems, Fuzzy Sets and Systems 20 (1) (1986) 31-43.

[13] M. Sakawa, H. Yano, T. Yumine, An interactive fuzzy satisficing method for multi objective linear-programming problems and its application, IEEE Transactions on Systems, Man and Cybernetics SMC 17 (1987) 654-661.

[14] S. Saminger, R. Mesiar, U. Bodenhofer, Domination of aggregation operators and preservation of transitivity, Internat. J. Uncertain. Fuzziness Knowledge-Based. Systems 10(Suppl.) (2002) 11-35.

[15] H.-J. Zimmermann, Fuzzy programming and linear programming with several objective functions, Fuzzy Sets and Systems 1 (1) (1978) 4555.
[16] H.-J. Zimmermann, Fuzzy Set Theory and Its Applications, Dordrecht, Boston, 1985. 